\title{
La era de los microorganismos multirresistentes: impacto en pacientes neutropénicos febriles
}

\author{
Recibido: 1/4/19 Aceptado: 9/7/19
}

Carolina Herrera1', Ezequiel Cordova', Wanda Cornistein², Viviana Oviedo, Nora Gómez³, Marcela Badía, Adolfo Wachs

\section{RESUMEN}

Introducción: El tratamiento antimicrobiano para los pacientes neutropénicos febriles (NF) se ha convertido en un desafío debido a la emergencia de microorganismos multirresistentes (MOR). El objetivo de este trabajo es analizar las características de estos pacientes y la incidencia de MOR.

Materiales y métodos: Estudio retrospectivo, observacional y descriptivo desde junio de 2015 hasta agosto de 2017 en adultos neutropénicos febriles hospitalizados en un hospital público de la ciudad de Buenos Aires. Se analizaron características demográficas, clínicas y microbiológicas, incluyendo los siguientes MOR: enterobacterias productoras de carbapenemasas (EPC) y beta-lactamasas de espectro extendido (BLEE), Acinetobacter baumannii complex, Enterococcus vancomicina resistente (EVR) y Stenotrophomonas maltophilia.

Resultados: Fueron incluidos 32 pacientes, 56\% mujeres con $84 \%$ de neoplasias hematológicas. Hubo colonización por EPC o EVR en el $59 \%$ de los pacientes. Se registraron 148 episodios infecciosos con $41 \%$ de documentación microbiológica. Los MOR fueron responsables del $25 \%$ de los episodios, siendo los más frecuentes Klebsiella pneumoniae productora de carbapenemasa y BLEE; los focos más frecuentes fueron bacteriemias e infecciones urinarias. Los pacientes con leucemias agudas (67\%) presentaron colonización por EPC o EVR en el $80 \%$. El tratamiento fue inadecuado en el $63 \%$ de las infecciones
${ }^{1}$ Unidad de Infectología. Hospital General de Agudos Dr. Cosme Argerich. CABA, Argentina.

${ }^{2}$ Servicio de Control de Infecciones. Hospital Universitario Austral.

${ }^{3}$ Laboratorio de Microbiología. Hospital General de Agudos Dr. Cosme Argeric". CABA, Argentina.

${ }^{4}$ Laboratorio central. Hospital General de Agudos Dr. Cosme Argerich. CABA, Argentina.

${ }^{5}$ Clínica médica. Hospital General de Agudos Dr. Cosme Argeric". CABA, Argentina.

Dirección para corespondencia: Carolina Herrera. Luis Agote 1670, Quilmes. Buenos Aires. CP: 1878.

Email: cccarohhherrera@gmail.com

Los contenidos del material no han sido publicados con anterioridad. Ninguno de los autores declara presentar conflicto de intereses en relación a esta publicación. por MOR y en el $12 \%$ por microorganismos sensibles (MS) $(p<0,01)$. La mortalidad global fue $53 \%$ con MOR y del $27 \%$ con MS ( $p=n s)$.

Conclusión: las infecciones por MOR fueron frecuentes con predominio de bacteriemias, especialmente EPC y BLEE. Por ello los MOR deben ser tenidos en cuenta para el tratamiento empírico en pacientes neutropénicos febriles.

Palabras clave: microorganismos multirresistentes, MOR, neutropénicos febriles, neoplasia. 


\section{Introducción}

En las últimas décadas se ha producido un cambio en el espectro microbiológico de las infecciones en pacientes neutropénicos febriles (NF) de causa oncológica. Existe una tendencia al desarrollo de bacterias multirresistentes a beta lactámicos habitualmente utilizados e, incluso, a la aparición de diferencias en estos patrones en función de las distintas áreas geográficas y locales (1).

Es así que este creciente desarrollo de microorganismos multirresistentes (MOR) se ha convertido en un grave problema sanitario a nivel mundial, con especial impacto en pacientes inmunosuprimidos.

Los MOR que generan infecciones en NF pueden agruparse bajo el acrónimo ESKAPES (Enterococcus faecium, Staphylococcus aureus, Klebsiella pneumoniae, Acinetobacter baumannii, Pseudomonas aeruginosa, Enterobacter spp., Escherichia coli, Stenotrophomonas maltophilia), siendo predominantes Klebsiella spp. y Escherichia coli en la mayoría de los estudios $(1,2)$.

Dada las limitadas opciones terapéuticas, los tratamientos para estos gérmenes son en general inadecuados, conllevando a elevadas tasas de morbi-mortalidad, sobre todo en pacientes con neoplasias hematológicas (3).

Si bien están bien descriptos los posibles factores de riesgo para la adquisición de MOR en NF $(1,2,3,4,5$, $6,7,8)$ es indispensable contar con información sobre la epidemiología, la distribución de los patógenos y los patrones de resistencia antimicrobiana locales dado que representan la piedra angular en el tratamiento empírico en este tipo de infecciones (1).

El objetivo del presente estudio es evaluar la epidemiología, los patrones de resistencia antimicrobiana, la evolución clínica y la incidencia de MOR en pacientes NF de causa oncológica para poder hallar estrategias que permitan adecuar el tratamiento empírico y disminuir su impacto negativo en este grupo particular de pacientes.

\section{Materiales y métodos}

Estudio retrospectivo, descriptivo y observacional realizado entre junio de 2015 y agosto de 2017 en un hospital público de tercer nivel de complejidad de la ciudad de Buenos Aires, Argentina. El hospital cuenta con 406 camas de internación, unidad de terapia intensiva y unidad coronaria para adultos.

Se registraron los episodios infecciosos de cada evento de neutropenia febril (ENF) de pacientes adultos mayores de 18 años internados con enfermedades onco-hematológicas. Todos se encontraban bajo tratamiento quimioterápico o en plan de iniciarlo en el caso de diagnóstico reciente.

Las variables analizadas fueron: edad, sexo, enfermedad de base, índice MASCC (Multinational Association for Supportive Care in Cancer), foco clínico de infección, recuento absoluto de neutrófilos, microorganismos aislados y su patrón de resistencia, tipo de tratamiento empírico y mortalidad a 30 días.

Para el estudio se consideraron las siguientes definiciones:

Evento de neutropenia febril: Periodo donde el paciente presentaba neutropenia y uno o más episodios febriles.

Episodio infeccioso: episodio donde el paciente neutropénico presentaba fiebre con sospecha de causa infecciosa. Estos se clasificaron en función de si tenían documentación microbiológica o no.

Neutropenia: Recuento de neutrófilos $<500 \mathrm{cel} / \mathrm{mm}^{3}$ o una caída esperada del recuento $<500 \mathrm{~mm}^{3}$ en las próximas 48 hs (9).

Fiebre: Temperatura axilar $>$ a $38,3^{\circ} \mathrm{C}$ o $>$ a $38^{\circ} \mathrm{C}$ sostenido en una hora (9).

Neutropenia funcional: Aquellas neoplasias oncohematológicas que producen neutrófilos defectuosos independientemente del recuento (9).

Neutropenia profunda: Recuento de neutrófilos $\leq 100 \mathrm{cel} /$ $\mathrm{mm}^{3}(9)$.

Neutropenia prolongada: Duración de la neutropenia mayor a 7 días (9).

Microorganismos multirresistentes (MOR): Bacterias con 
resistencia mayor o igual a tres familias de antimicrobianos diferentes (4). Se incluyeron: Staphylococcus aureus meticilino resistente; Pseudomonas aeruginosa resistente a penicilinas antipseudomónicas, cefalosporinas, carbapenems y quinolonas; Stenotrophomonas maltophilia; Enterococcus spp. resistente a vancomicina (EVR); Acinetobacter baumannii complex (ABA) y enterobacterias productoras de betalactamasa de espectro extendido (BLEE) o de carbapenemasas (EPC)

Tratamiento inadecuado: Droga administrada sin actividad in vitro contra la bacteria responsable de la infección de acuerdo a los resultados de los test de sensibilidad antimicrobiana (4)

Mortalidad a 30 días: Muerte dentro de los 30 días del inicio de la infección documentada.

Durante el período de estudio se realizaron hisopados rectales de vigilancia semanales en búsqueda de EVR y enterobacterias productoras de carbapenemasas (EPC).

\section{Estudio microbiológico}

La determinación de la sensibilidad antimicrobiana in vitro se efectuó mediante los métodos de difusión por disco, E-test o por el sistema automatizado VITEK 2 (BioMerieux). Se utilizaron los criterios y normativas nacionales de la red WHONET Argentina para determinar la sensibilidad a los agentes antimicrobianos (15).

\section{Resultados}

\section{Características demográficas y clínicas}

Durante el periodo de estudio se incluyeron 32 pacientes, se identificaron 81 eventos de NF y 148 episodios infecciosos. Las características de los pacientes, los episodios infecciosos y los patógenos causales están descriptos en las Tablas 1, 2 y 3.

La edad media de los pacientes fue de 53 años (rango 18-75); de ellos, $84 \%$ tenían neoplasias hematológicas (leucemias agudas en el $56 \%$ ) y $16 \%$ tumores sólidos. La media de internación por paciente fue de 2 días (DS \pm 1.4$)$. Se registró colonización por EPC y/o EVR en el $59 \%$ de los pacientes en algún momento de su internación.

Los pacientes presentaron neutropenia profunda en el $37 \%(55 / 148)$ de los episodios infecciosos y neutropenia
Tabla 1. Características clínico-demográficas de los pacientes estudiados

\begin{tabular}{|l|l|}
\multicolumn{1}{c|}{ Variables } & \multicolumn{1}{c|}{ n (\%) } \\
\hline $\begin{array}{l}\text { Número de pacientes } \\
\text { Edad (mediana) }\end{array}$ & 32 \\
\hline Sexo femenino & $18(56 \%)$ \\
\hline $\begin{array}{l}\text { Neoplasias sólidas } \\
\text { Neoplasias hematológicas }\end{array}$ & $5(16 \%)$ \\
\hline Leucemias agudas (LMA, LLA) & $27(84 \%)$ \\
\hline Linfoma de Hodgkin & $15(56 \%)$ \\
\hline $\begin{array}{l}\text { Linfoma difuso de células B } \\
\text { grandes } \\
\text { Otros }\end{array}$ & $3(11 \%)$ \\
\hline Mediana días de internación & $3(11 \%)$ \\
\hline
\end{tabular}

\section{Tabla 2. Características de los episodios} infecciosos

\begin{tabular}{|c|c|}
\hline Variables & n (\%) \\
\hline Episodios de neutropenia febril & 81 \\
\hline Episodios infecciosos & 148 \\
\hline $\begin{array}{l}\text { Con documentación } \\
\text { microbiológica }\end{array}$ & $60(41)$ \\
\hline $\begin{array}{l}\text { Sin documentación } \\
\text { microbiológica }\end{array}$ & $88(59)$ \\
\hline Microorganismos aislados & $77(100)$ \\
\hline $\begin{array}{l}\text { Microorganismos } \\
\text { multirresistentes (MOR) }\end{array}$ & 19/77 (25) \\
\hline Episodios infecciosos con DM & $60(100)$ \\
\hline $\begin{array}{l}\text { Episodios infecciosos con DM y } \\
\text { MOR }\end{array}$ & $16 / 60(27)$ \\
\hline
\end{tabular}

MOR: microorganismos multirresistentes DM: documentación microbiológica 


\begin{tabular}{|c|c|c|}
\hline Microorganismos & n (\%) & n MOR (\%) \\
\hline 1. Bacilos Gram negativos & \multicolumn{2}{|r|}{$35(45)$} \\
\hline Klebsiella pneumoniae & $18(51)$ & \\
\hline Escherichia coli & $7(20)$ & $\begin{array}{l}\text { KPC } 9 / 35 \\
(26)\end{array}$ \\
\hline Enterobacter spp. & $4(11)$ & $\begin{array}{l}\text { BLEE 5/35 } \\
(14)\end{array}$ \\
\hline Serratia spp. & $4(11)$ & \\
\hline Otros & $2(7)$ & \\
\hline 2. Cocos Gram positivos & \multicolumn{2}{|r|}{$14(18)$} \\
\hline $\begin{array}{l}\text { Staphylococcus coagulasa } \\
\text { negativa }\end{array}$ & $6(43)$ & \multirow{3}{*}{ EVR 1/14 (7) } \\
\hline Enterococcus spp. & $4(29)$ & \\
\hline Streptococcus spp. & $4(29)$ & \\
\hline $\begin{array}{l}\text { 3. Bacilos Gram negativos } \\
\text { no fermentadores }\end{array}$ & \multicolumn{2}{|r|}{$6(10)$} \\
\hline Acinetobacter spp. & $4(66)$ & \\
\hline$P$. aeruginosa & $1(17)$ & ABA $3 / 6(50)$ \\
\hline S. maltophilia & $1(17)$ & $\begin{array}{l}\text { S. maltophilia } \\
1 / 6(17)\end{array}$ \\
\hline 4. Toxina para Clostridium difficile & \multicolumn{2}{|r|}{$11(14)$} \\
\hline 5. Hongos & \multicolumn{2}{|r|}{$8(10)$} \\
\hline 6. Otros & & $3(4)$ \\
\hline
\end{tabular}

KPC: Klebsiella productora de carbapenemasa BLEE: betalactamasa de espectro extendido ABA: Acinetobacter baumanii

EVR: Enterococcus vancomicina resistente
Tabla 4. Descripción de los

microorganismos documentados según foco clínico de infección

\begin{tabular}{|c|c|c|}
\hline Foco infeccioso & n (\%) & n (\%) MOR \\
\hline 1. MD & $\begin{array}{l}77 \\
(100)\end{array}$ & \\
\hline 2. Bacteriemias & $33(43)$ & \\
\hline K. pneumoniae & $10(30)$ & $\begin{array}{l}\text { KPC: } 3(30) \text { y } \\
\text { BLEE: } 3(30)\end{array}$ \\
\hline $\begin{array}{l}\text { Staphylococcus coagulasa } \\
\text { negativa }\end{array}$ & $5(16)$ & \\
\hline Acinetobacter spp. & $3(9)$ & ABA:3 (67) \\
\hline Escherichia coli & $3(9)$ & \\
\hline Streptococcus spp. & $3(9)$ & \\
\hline Enterococcus spp. & $3(9)$ & EVR: 1 (33) \\
\hline Enterobacter spp. & $2(6)$ & \\
\hline Serratia spp. & $2(6)$ & \\
\hline Otros & $2(6)$ & \\
\hline $\begin{array}{l}\text { 3. Infecciones del tracto urinario } \\
\text { (ITU) }\end{array}$ & $14(18)$ & \\
\hline K. pneumoniae & $5(36)$ & $\begin{array}{l}3 \text { (60) KPC y } 1 \\
\text { (20) BLEE }\end{array}$ \\
\hline Escherichia coli & $4(29)$ & \\
\hline Enterococcus spp. & $4(29)$ & \\
\hline Acinetobacter baumanii & $1(7)$ & \\
\hline 4. Pleuro pulmonar & $7(9)$ & 1 (14) KPC \\
\hline $\begin{array}{l}\text { 5. Abdominal (toxina Clostridium } \\
\text { difficile positiva) }\end{array}$ & $11(14)$ & \\
\hline 6. Sinusopatías & $4(5)$ & \\
\hline 7. IPPB & $2(3)$ & \\
\hline 8. IAC & $3(4)$ & \\
\hline 9. Otros & $3(4)$ & \\
\hline
\end{tabular}

MD: microorganismo documentado IPPB: infección de piel y partes blanda IAC: infección asociada a catéter EPC: enterobacterias productores de carbapenemasas.

BLEE: beta- lactamasa de espectro extendido ABA: Acinetobacter baumannii complex EVR: Enterococcus vancomicina resistente. KPC: Klebsiella productora de carbapenemasa 
prolongada en el 46,6\% (69/148). La duración media de la neutropenia en los eventos infecciosos fue de 10 días (DS $\pm 12,5)$.

Con respecto a los microorganismos documentados ( $\mathrm{n}=77$ ), el $45 \%$ fueron bacilos Gram negativos (BGN) con predominio de enterobacterales $(44 \%, \mathrm{n}=34)$, seguidos de cocos Gram positivos $(18 \%, \mathrm{n}=14)$ y bacterias Gram negativas no fermentadoras $(8 \%, n=6)$ (ver Tablas 3 y 4$)$.

El patógeno más frecuente fue Klebsiella pneumoniae ( $23 \%$, $\mathrm{n}=18)$, seguido de Escherichia coli $(9 \%, \mathrm{n}=7)$ y Staphylococcus coagulasa negativa $(8 \%, n=6)$. El $40 \%$ de las infecciones por BGN fueron por KPC y BLEE. A nivel global las infecciones más frecuente fueron las bacteriemias y las del tracto urinario, sobre todo por K. pneumoniae (Ver Tabla 4).

\section{Características de los MOR}

Quince pacientes $(15 / 32 ; 47 \%)$ desarrollaron infecciones por MOR; éstos representaron el $25 \%$ de todos los microorganismos documentados (19/77). Los MOR más frecuentes fueron las enterobacterias, en especial Klebsiella pneumoniae KPC $(9 / 19 ; 47 \%)$ y BLEE $(5 / 19 ; 26 \%)$ seguido de $A$. baumannii (3/19; $16 \%)$, EVR $(1 / 19 ; 5 \%)$ y $S$. maltophilia $(1 / 19 ; 5 \%)$ (ver Tabla 3$)$.

Las bacteriemias fueron el tipo de infección más frecuente (47\%), con rescates de MOR en $27 \%$ junto con las del tracto urinario (26\%) (ver Tabla 5).

La media de días de internación desde la admisión hasta el desarrollo de infecciones por MOR fue de 42. Estos pacientes presentaron internación prolongada (media de 61 días), colonización por EPC o EVR en algún momento de su internación $(80 \%, 12 / 15)$ y leucemias agudas como patología de base (67\%).

Con respecto a la resistencia antibiótica acompañante, se pudieron determinar las siguientes características de 18 de los 19 MOR: KPC con resistencia a colistin $62,5 \%(5 / 8)$ y amikacina $37,5 \%(3 / 8)$ con sensibilidad a tigeciclina del $100 \%$ (8/8); ABA resistente a carbapenémicos 100\% (3/3), tigeciclina $33 \%(1 / 3)$ sin resistencia a colistin o amikacina. Las enterobacterias productoras de BLEE fueron resistentes a ciprofloxacina $100 \%(5 / 5)$, piperacilina tazobactam $60 \%$ (3/5) y amikacina $20 \%(1 / 5)$, conservando sensibilidad frente a colistin en el $100 \%(5 / 5)$. S. maltophilia fue sensible

\section{Tabla 5. Focos infecciosos de los MOR*}

\begin{tabular}{|l|l|l|}
\hline \multicolumn{1}{|c|}{ Focos clínicos } & $\begin{array}{l}\mathrm{y}(\%) \text { MOR } \\
\mathrm{n}(\%) \\
\text { documentado } \\
\text { KPC 3 (33) } \\
\text { Bacteriemias }\end{array}$ & $\begin{array}{l}\text { BLEE 3 (33) } \\
\text { ABA 2 (22) } \\
\text { EVR 1 (11) } \\
\text { KPC 3 (60) } \\
\text { BLEE 1 (20) } \\
\text { ABA 1 (20) }\end{array}$ \\
\hline Tracto urinario & $5(26)$ & $\begin{array}{l}\text { KPC 1 (50) } \\
\text { S. maltophilia } \\
1(50)\end{array}$ \\
\hline Pleuro pulmonar & $2(11)$ \\
\hline Otros & $3(16)$ & \\
\hline
\end{tabular}

*n total de MOR: 19

a trimetoprima sulfametoxazol, levofloxacina y minociclina y EVR a linezolid, quinupristina y dalfopristina.

Todos los aislamientos de ABA y KPC presentaron resistencia a meropenem, con una $\mathrm{CIM}>=16$ en todos los casos.

\section{Tratamiento y evolución clínica}

Todos pacientes recibieron tratamiento antimicrobiano en función de la sospecha clínica inicial. Los antibióticos más utilizados incluyeron piperacilina tazobactam $(17 \% ; 25 / 148)$ y carbapenémicos (78\%; 115/148). La combinación antibiótica más empleada fue meropenem y colistin (43\%; 50/115).

Los esquemas antibióticos empíricos fueron inadecuados en el $63 \%$ de las infecciones por MOR (12/19) y en el $12 \%$ de las infecciones por microorganismos sensibles $(7 / 58)$ $(p=0,0001)$

Sesenta de los 148 episodios infecciosos (60/148) tuvieron documentación microbiológica; los MOR se aislaron en 16 de estos $(16 / 60)$.

Con respecto a los episodios infecciosos con MOR (16/60), se detectaron $81 \%$ de colonizaciones positivas (13/16) mientras que fue de $45 \%$ (20/44) en episodios con gérmenes multisensibles $(p=0,02)$ (ver Tabla 2). 
La mortalidad global a 30 días fue del $45 \%$ (15/32), con una mortalidad de $53 \%(8 / 15)$ en pacientes con MOR y de $27 \%$ $(4 / 11)$ con microorganismos sensibles $(p=0,45)$.

\section{Discusión}

Las infecciones por MOR en pacientes neutropénicos febriles de causa onco-hematológica han aumentado considerablemente su incidencia en el último tiempo (4).

En la población analizada, el $25 \%$ de los episodios infecciosos con documentación microbiológica fueron asociados a MOR con predominio de bacilos Gram negativos, en especial Klebsiella pneumoniae en coincidencia con la literatura $(1,4)$.

Tanto para MOR como para microorganismos sensibles, las infecciones más frecuentes fueron las bacteriemias y las del tracto urinario, en concordancia con otros trabajos publicados previamente $(1,4)$. Los MOR fueron responsables del $24 \%$ de las bacteriemias y el $36 \%$ de las infecciones urinarias $(1,4)$, siendo los agentes etiológicos más frecuentes $K$. pneumoniae tipo KPC, BLEE y A. baumannii complex.

En diversos estudios se describen los posibles factores de riesgo para MOR en neutropénicos febriles, como el estado de inmunosupresión, la exposición previa a ATB de amplio espectro (en especial dentro del mes previo), la presencia de comorbilidades, la tenencia de catéter urinario, las infecciones nosocomiales, la hospitalización prolongada o repetida, edad avanzada, internación en terapia intensiva y colonización por MOR previa $(1,2,4,6,7,8)$. En el caso de los pacientes estudiados se pudo observar que aquellos con infecciones por MOR presentaban internación prolongada, neutropenias profundas y colonización por EPC o EVR en algún momento de su internación.

Las infecciones por gérmenes multirresistentes suelen asociarse a peor pronóstico y elevada mortalidad (cercana al $50 \%-67 \%$ según diferentes estudios) $(4,10,11,12)$. En nuestra serie, la mortalidad fue elevada, aunque en comparación con las muertes en pacientes con bacterias sensibles no fue estadísticamente significativo.

Dadas las escasas opciones terapéuticas disponibles para tratar los MOR, en general el tratamiento empírico suele ser inadecuado, como se ha visto en nuestro trabajo. Por esta situación existen varias estrategias propuestas en la literatura. Una de ellas, sugerida por The European Conference on Infections in Leukemia (13), implica iniciar una terapia empírica "descalativa" de antibióticos en pacientes NF con alto riesgo de desarrollar infecciones por EPC. En función de la presencia de factores de riesgo para bacterias resistentes $y$ de cursos clínicos complicados se deberían incluir dos drogas activas en base a la epidemiología y resistencia locales. Si los cultivos son negativos o los gérmenes son sensibles se debe decalar el tratamiento inicial $(10,13,14)$.

Nuestro estudio presenta algunas limitaciones, como ser retrospectivo y con un número pequeño de pacientes, por lo cual las conclusiones no pueden ser generalizadas. Sin embargo, creemos que los resultados pueden ser de sumo valor para guiar las decisiones terapéuticas en este grupo de pacientes de riesgo.

A modo de conclusión, las infecciones por MOR han resultado ser frecuentes en la población estudiada, con gran predominancia de BGN, especialmente las enterobacterias KPC y BLEE. Dada la elevada mortalidad y peor pronóstico de estas infecciones es importante tener en cuenta estos MOR a la hora de desarrollar estrategias de tratamiento empírico en pacientes neutropénicos febriles. 


\section{Referencias}

1. Rapoport B, Klastersky J, Raftopoulos H, Freifeld A, Aoun $M$, Zinner $S$ et. al. The emerging problem of bacterial resistance in cancer patients; proceedings of a workshop held by MASCC "Neutropenia, Infection and Myelosuppression" Study Group during the MASCC annual meeting held in Berlin on 27-29 June 2013. Support Care Cancer. 2016; 24:2819-2826.

2. Esposto A, Aguilera K, Lizaso D, Ferrer F, Nuccetelli $\mathrm{Y}$, Yantorno $\mathrm{M}$ et. al. Bacteriemias por bacilos Gram negativos multirresistentes en neutropénicos febriles con enfermedades hematológicas. Actualizaciones en SIDA. 2013; 21.81: 73-83.

3. Micozzi A, Gentile G, Minotti C, Cartoni C, Capria S, Ballarò D et. al. Carbapenem-resistant Klebsiella pneumoniae in high-risk haematological patients: factors favouring spread, risk factors and outcome of carbapenem resistant Klebsiella pneumoniae bacteremias. BMC Infect Dis. 2017; 17(1):203.

4. Bastug A, Kayaaslan B, Kazancioglu S, But A, Aslaner H, Akinci $E$ et. al. Emergence of multidrug resistant isolates and mortality predictors in patients with solid tumors or hematological malignancies. J Infect Dev Ctries. 2015; 9(10):1100-1107.

5. Gudiol C, Tubau F , Calatayud L , Garcia-Vidal C, Cisnal M, Sánchez-Ortega I et.al. Bacteraemia due to multidrugresistant Gram-negative bacilli in cancer patients: risk factors, antibiotic therapy and outcomes. J Antimicrob Chemother. 2011; 66(3):657-63.

6. Bossaer J, Hall P, Garrett-Mayer E. Incidence of vancomycin-resistant enterococci (VRE) infection in high-risk febrile neutropenic patients colonized with VRE. Support Care Cancer. 2010; 19(2):231-7.

7. Zaas A, Song X, Tucker P, Perl T. Risk factors for development of vancomycin-resistant enterococcal bloodstream infection in patients with cancer who are colonized with vancomycin-resistant enterococci. Clin Infect Dis. 2002; 35(10):1139-46.

8. Ford C, Lopansi BK, Haydoura S, Snow G, Dascomb $\mathrm{KK}$, Asch J et. al. Frequency, risk factors, and outcomes of vancomycin-resistant Enterococcus colonization and infection in patients with newly diagnosed acute leukemia: different patterns in patients with acute myelogenous and acute lymphoblastic leukemia. Infect Control Hosp Epidemiol. 2015; 36(1):47-53.

9. Alison G, Freifeld E, Bow J, Sepkowitz A, Michael J, Boeckh $J$ et. al. Clinical Practice Guideline for the Use of Antimicrobial Agents in Neutropenic Patients with Cancer: 2010 Update by the Infectious Diseases Society of America. Clinical Infectious Diseases. 2013; 52 (4): 56-93.

10. Tofas P,Skiada A, Angelopoulou M, Sipsas N, Pavlopoulou I, Tsaousi S et. al. Carbapenemase-producing Klebsiella pneumonia bloodstream infections in neutropenic patients with haematological malignancies or aplastic anaemia: analysis of 50 case. Int $\mathrm{J}$ Antimicrob Agents. 2016; 47(4):335-9.

11. Girmenia C, Viscoli C, Piciocchi A, Cudillo L, Botti S, Errico A et. al. Management of carbapenem resistant klebsiella pneumonia infections in stem cell transplant recipients: an Italian multidisciplinary consensus statement. Haematologica. 2015; 100 (9): 373-376.

12. Righi E, Peri AM, Harris $P$, Wailan AM, Liborio M, Lane $S$ et .al. Global prevalence of carbapenem resistance in neutropenic patients and association with mortality and carbapenem use: systematic review and meta-analysis. J Antimicrob Chemother. 2017; 72 (3): 668-677.

13. Averbuch D, Orasch C, Cordonnier C, Livermore D, Mikulska M, Viscoli C et. al. European guidelines for empirical antibacterial therapy for febrile neutropenic patients in the era of growing resistance: summary of the 2011 4th European Conference on Infections in Leukemia. Haematologica. 2013; 98 (12): 1826-1835.

14. Sehnaz A, Murat A. Management of febrile neutropenia in the era of bacterial resistance. Ther Adv Infect Dis. 2013; 1 (1): 3743

15. Red WHONET-Argentina. Protocolo de Trabajo acordado en el VXII Taller WHONET-Argentina" 2016. Disponible en http://antimicrobianos.com.ar/2014/10/protocolo-detrabajo-red-whonet-argentina/ 
The era of multidrug-resistant microorganisms: impact on febrile neutropenic patients

Background: Antimicrobial treatment for febrile neutropenic (FN) patients has become a challenge due to the growing emergence of multidrug-resistantmicroorganisms(MDR-MO). The objective of this study was to analyze the characteristics of these population and the incidence of MDR-MO. Methods \& Materials: Retrospective, observational and descriptive study from June 2015 to August 2017 in FN adults hospitalized at a public hospital in Buenos Aires city, Argentina. Demographic, clinical and microbiological characteristics were analyzed. We included the following MDR-MO: extended spectrum beta-lactamase (ESBL) and carbapenemase-producing Enterobacteriaceae (CPE), Acinetobacter baumannii complex, vancomycin resistant Enterococcus (VRE) and Stenotrophomonas maltophilia. Results: Thirty-two patients were included; $56 \%$ were women, with $84 \%$ haematological diseases. Colonization by CPE or VRE was observed in a $59 \%$ of the patients. There were 148 infectious episodes. Of them $41 \%$ had microbiological documentation. MDR-MO were responsible for $25 \%$ of the episodes and the most frequent were carbapenemase-producing Klebsiella pneumoniae and ESBL producing Enterobacteriaceae. MDR-MO were isolated mainly from bacteremia and urinary infections, patients had acute leukemia in a $67 \%$ and colonization CPKP or VRE in $80 \%$. Inadequate treatment for MDR-MO was observed in $63 \%$ of the cases and $12 \%$ for susceptible microorganisms $(p<0,01)$. The mortality was $53 \%$ for MDR-MO and $27 \%$ for susceptible microorganisms ( $p=n s)$. Conclusion: MDR-MO infections were frequent with predominance of bacteremia especially CPE and ESBL producing Enterobacteriaceae. According to these results MDR-MO should be taken into account for the empiric antimicrobial treatment in febrile neutropenic patients.

Keywords: Multidrug resistant microrganisms, MDR-MO, febrile neutropenic, neoplasic. 